Herzschr Elektrophys 2018 $29: 349-354$ https://doi.org/10.1007/s00399-018-0593-8 Eingegangen: 30. Juli 2018

Angenommen: 10. September 2018

Online publiziert: 10. Oktober 2018

(c) Der/die Autor(en) 2018

CrossMark

Philipp Niehues - Gerrit Frommeyer · Florian Reinke · Lars Eckardt

Klinik für Kardiologie II - Rhythmologie, Universitätsklinikum Münster, Albert-Schweitzer Campus 1, Münster, Deutschland

\title{
Subkutan implantierbarer Kardioverter-Defibrillator
}

\section{Aktueller Stand und Ausblick}

Implantierbare Defibrillatorsysteme sind seit Jahren fester Bestandteil in der Primär- und Sekundärprophylaxe des plötzlichen Herztods, ihr Nutzen ist durch zahlreiche randomisierte Studien belegt. Neben den herkömmlichen transvenösen Systemen steht seit 2009 ein rein subkutanes implantierbares Kardioverter-Defibrillator-System (S-ICD, Cameron Health, San Clemente, CA, USA; jetzt Boston Scientific, USA) zur Verfügung, das aufgrund des reduzierten Risikos für Elektrodendysfunktion und systemische Infektionen eine gute und zunehmend häufiger genutzte Alternative für bestimmte Patientenkollektive darstellt. Erste große Registerstudien untermauern Sicherheit und Effektivität. Andererseits kommt das subkutane System aufgrund limitierter Therapiefunktionen nicht für jeden in Betracht. Perspektivisch scheint die Eignung für ein breiteres Patientenkollektiv unseres Erachtens aber realistisch.

Im Rahmen der stetigen Weiterentwicklung implantierbarer Defibrillatorsysteme gibt es durch den subkutanen ICD seit nunmehr fast 10 Jahren die Möglichkeit, im individuellen Fall auf die Implantation transvenöser Elektroden zu verzichten. Eine sichere Arrhythmiedetektion und -terminierung auch ohne transvenöse Elektrode konnte initial bereits an einem relativ kleinen Kollektiv von 55 Patienten durch die Zulassungsstudie gezeigt werden [1]. Mehr als 4000 Patienten wurden seitdem in teils noch laufende Studien eingeschlossen, um die Sicherheit und Effektivität des S-ICD zu überprüfen. Seit 2015 befindet sich das Gerät der 2. Generation, der EM-
BLEM $^{\mathrm{TM}}$ S-ICD (Modell A209), auf dem Markt. Seit 2016 ist dieser auch in einer MRT-fähigen Version (EMBLEM ${ }^{\mathrm{TM}}$ MRI S-ICD) verfügbar. Die nicht in Deutschland vertriebene Version EMBLEM $^{\mathrm{sm}}$ MRI (Modell A219) ist zusätzlich zur Detektion und Therapie ventrikulärer Episoden in der Lage, auftretende Vorhofflimmerepisoden $\mathrm{zu}$ detektieren und zu speichern. Obwohl die Zahl der Implantationen von Jahr zu Jahr zunimmt, lag der Anteil an S-ICD-Implantationen in Deutschland im Jahr 2015 bei lediglich 2,3\% [2]. Insbesondere im Hinblick auf die mit der rechtsventrikulären ICD-Elektrode assoziierten perioperativen Akutkomplikationen (Pneumothorax, Perikardtamponade etc.) sowie möglicher Probleme im Langzeitverlauf (systemische Infektionen, Elektrodendysfunktion; [3]) scheint der S-ICD durch seine rein subkutane Lage im Vergleich zu transvenösen Systemen entscheidende Vorteile zu bieten, zumal systemische Infektionen mit einem erhöhten Mortalitätsrisiko einhergehen [4]. In der Vergangenheit konnte gezeigt werden, dass 8 Jahre nach Implantation nur noch ca. 60-70\% der transvenös eingebrachten Elektroden funktionstüchtig sind [5]. In diesem Zusammenhang ist das nicht $\mathrm{zu}$ vernachlässigende Risiko einer Elektrodenextraktion zu berücksichtigen.

Entscheidender Nachteil der S-ICDTherapie ist, dass das Gerät bis dato nicht in der Lage ist, antitachykarde Stimulation im Sinne von Abgaben zum antitachykarden Pacing (ATP) bzw. antibradykarde Stimulation zu ermöglichen. Es bie- tet lediglich die Möglichkeit einer PostSchock-Stimulation für $30 \mathrm{~s}$ (s. unten).

\section{Implantation und Funktionsweise}

Bezüglich des Vorgehens bei operativer Implantation hat sich seit Einführung des S-ICD nichts Wesentliches verändert. Weiterhin erfolgt der Eingriff meist in Intubationsnarkose, wobei der Generator des Systems linksseitig in Höhe des 5. bis 6. Interkostalraums in der mittleren Axillarlinie subkutan positioniert wird. Die Elektrode mit 2 Wahrnehmungspolen und einer dazwischen liegenden Schockwendel wird anschließend in 2 Schritten zunächst bis zum Xiphoid und dann meist parasternal links oder, je nach Herzkonfiguration und -lage, alternativ auch rechts parasternal subkutan [6] bis ungefähr zum Beginn des Manubriums getunnelt, bevor sie mit dem Aggregat verbunden wird. Da anatomische Strukturen als Orientierung bei der Implantation verwendet werden, kann intraoperativ auf eine röntgenmorphologische Bildgebung verzichtet werden, wobei ein präoperatives Röntgenbild zur Bestimmung der Herzlage und zur Operationsplanung [6] Standard sein sollte. Nach Implantation erfolgt das Setup, bei dem das System automatisch auf Grundlage der R-Zacken-Amplitude, des R-Zacken/T-Wellen-Verhältnisses und der geringsten Störsignale den besten von 3 zur Verfügung stehenden Wahrnehmungsvektoren (primär, sekundär, alternativ) auswählt (• Abb. 1). Der automatische Test wird zunächst im Liegen und im Anschluss an die 


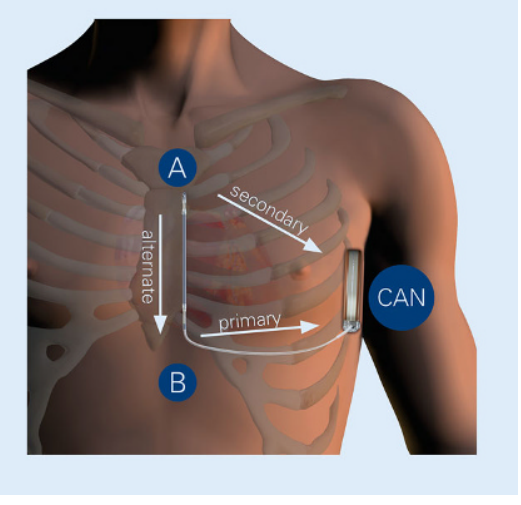

Abb. 1 ^ Vektoren zur Wahrnehmung (weiß). Primärer Vektor: $B>C A N$. Sekundärer Vektor: $A>C A N$. Alternativer Vektor: $A>B$. (Abdruck mit freundl. Genehmigung von Boston Scientific)

Operation auch in sitzender Position durchgeführt. Außerdem sollte unter körperlicher Ausbelastung (z. B. mittels Fahrradergometrie) eine fehlerhafte Detektion bzw. ein T-Wellen-Oversensing ausgeschlossen werden [7]. Im Verlauf kann dieser Warnehmungsvektor beispielsweise bei Auftreten von Oversensing automatisch oder manuell umprogrammiert werden. In einer kürzlich publizierten Studie wurde ein Wechsel des Wahrnehmungsvektors bei 35,7\% der Patienten beobachtet. Hierdurch konnte in Einzelfällen das Auftreten inadäquater Therapieabgaben vermieden werden, wenngleich der Wechsel des Wahrnehmungsvektors im Gesamtkollektiv keine inadäquaten Therapieabgaben verhinderte [8]. Diese Daten belegen jedoch, dass nach Möglichkeit mehrere Wahrnehmungsvektoren bei Implantation programmierbar sein sollten. Im Gegensatz zu konventionellen transvenösen Systemen sollte am Ende jeder Operation standardmäßig eine ICD-Testung durchgeführt werden. Dabei erfolgt zunächst die Induktion von Kammerflimmern mittels eines 50-Hz-Bursts, bevor das Gerät nach entsprechender Arrhythmiedetektion einen 65-J-Schock abgibt. Dabei wird im Vergleich zur standardmäßigen Energieabgabe von $80 \mathrm{~J}$ eine Sicherheitsmarge von $15 \mathrm{~J} \mathrm{kal}$ kuliert. Dass die Testung des subkutanen ICD-Systems nicht unterlassen werden sollte, zeigte u. a. eine 2016 durchgeführ-
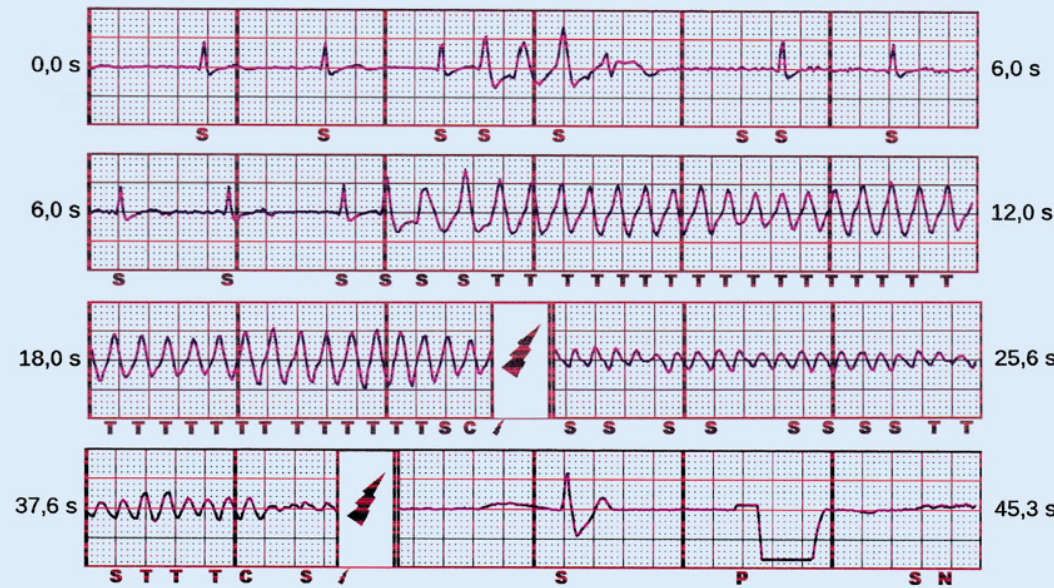

Abb. 2 \ Subkutanes Elektrokardiogramm: regelrechte Detektion von Kammerflimmern mit Terminierung durch die zweite ICD-Schockabgabe. Anschließend einmaliges Postschock-Pacing. ICD implantierbarer Kardioverter-Defibrillator

te prospektive Registerstudie. Hier war bei 102 Implantationen die erste Testung lediglich in $75 \%$ der Fälle erfolgreich. Durch Änderung der Schockpolarität, Repositionierung von Elektrode oder Aggregat bzw. Programmierung der Schockenergie auf $80 \mathrm{~J}$ konnte in den darauffolgenden Testungen in jedem Fall eine interne VF-Terminierung erreicht werden [9]. Für den klinischen Gebrauch sind lediglich 2 Detektionszonen programmierbar, wobei in der sog. bedingten Schockzone, anders als in der Schockzone, neben der Frequenz auch Faktoren wie ein aufgezeichneter Referenzkomplex und die QRSBreite zum Erkennen behandlungsbedürftiger Arrhythmien genutzt wird. Die Schockzone ist in 10er-Intervallen zwischen 170 und 250 Schlägen pro Minute programmierbar. Unter Berücksichtigung der Ergebnisse der MADITRIT-Studie [10], die eine Reduktion von Mortalität und inadäquaten ICDTherapien durch zurückhaltende Programmierung nahelegt, programmieren die Autoren die bedingte Schockzone standardmäßig ab einer Frequenz von 220/min und die Schockzone ab einer Frequenz von 240/min. Das Gerät ist in der Lage, 5 konsekutive synchronisierte Schocks pro Episode abzugeben. Dabei erfolgt der biphasische Schockimpuls zwischen Generator und Schockwendel, wobei die Polarität des ersten Schocks („standard“ vs. „reversed“) program- mierbar ist. Bei Ineffektivität der ersten Schockabgabe wird die Schockpolarität automatisch für jeden weiteren Schock umgekehrt (-Abb. 2). Ist ein Schock erfolgreich, so wird dessen Polarität automatisch als Ausgangspolarität für folgende Episoden programmiert. Nach erfolgreicher Schockabgabe ist das Gerät optional in der Lage, über einen Zeitraum von 30 s eine Post-Schock-Stimulation mit einer Frequenz von 50/min zu ermöglichen. Laut Hersteller beträgt die durchschnittliche Funktionsdauer der aktuellen Modelle bis zur Batterieerschöpfung 7,3 Jahre. Die Autoren konnten kürzlich nachweisen, dass bei den ersten 28 Patienten, die in ihrem Zentrum einen subkutanen ICD des ersten Modells „1010“ erhielten, der erste Aggregatwechsel nach 6,3 $\pm 0,5$ Jahren durchgeführt wurde [11].

\section{S-ICD-Screening}

Vor Implantation eines subkutanen ICDSystems ist ein Screening jedes individuellen Patienten entscheidend, um Signaleigenschaften zu identifizieren, die eine sichere Detektion erschweren bzw. verhindern. Einerseits steht hierfür seit Kurzem das sog. Automated Screening Tool (AST) zur Verfügung, bei dem mittels des Programmiergeräts und speziell geklebten EKG-Elektroden alle 3 Wahrnehmungsvektoren für eine linksparasternale sowie rechtsparasternale Elektrodenla- 
ge getestet werden können. Alternativ ist ein manuelles Screening (MST) mittels speziellen Schablonen und OberflächenEKGs möglich. Die 2 Screening-Tools dienen dem gleichen Zweck und können unabhängig voneinander oder zusammen verwendet werden. Beide weisen eine ähnliche Sensitivität auf, wobei der standardisierte Prozess beim automatischen Screening-Tool vorteilhaft erscheint [12].

\section{Welches Patientenkollektiv kommt infrage?}

Die Empfehlungen der aktuellen Leitlinien der European Society of Cardiology (ESC) aus dem Jahr 2015 sowie der American Heart Association (AHA) aus dem Jahr 2017 basieren auf den individuellen Vor- und Nachteilen des S-ICD-Systems. Beide empfehlen den S-ICD als Alternative zum transvenösen System, wenn keine Indikation für eine intrakardiale Stimulation im Sinne einer antibradykarden Therapie, ATP-Abgabe oder kardialen Resynchronisationstherapie besteht oder zu einem späteren Zeitpunkt zu erwarten ist (Empfehlungsgrad IIa; $[13,14])$. Bezüglich eines zu erwartenden Stimulationsbedarfs legen einige Studien aus den letzten Jahren gewissen Prädiktoren nahe. Hierzu zählen im Besonderen ein breiter QRS-Komplex, eine verlängerte PQ-Zeit sowie ein NYHA-Stadium III oder IV [15]. Besonders die fehlende Möglichkeit zur Abgabe programmierbarer antitachykarder Stimulation (ATP) führt dazu, dass sich einige Patientenkollektive mehr als andere für die Implantation eines subkutanen ICD eignen. So sind Patienten, bei denen das Auftreten monomorpher ventrikulärer Tachykardien aufgrund ihrer Grunderkrankung nicht zu erwarten ist, in der Regel nicht auf eine ATP-Abgabe angewiesen. Hierzu zählen im Besonderen Patienten mit primär elektrischen Herzerkrankungen, für die das Risiko eines plötzlichen Herztods durch primäres Kammerflimmern begründet ist. Für Patienten, die zusätzlich zu den schon genannten Eigenschaften ein hohes Infektionsrisiko bei transvenösem Zugang aufweisen bzw. bei denen das transvenöse Einbringen einer Elektrode aufgrund von anatomischen

Herzschr Elektrophys 2018 · 29:349-354 https://doi.org/10.1007/s00399-018-0593-8

(c) Der/die Autor(en) 2018

P. Niehues · G. Frommeyer · F. Reinke · L. Eckardt

\section{Subkutan implantierbarer Kardioverter-Defibrillator. Aktueller Stand und Ausblick}

\section{Zusammenfassung}

Der Nutzen implantierbarer Defibrillatorsysteme zur Prävention des plötzlichen Herztods ist durch zahlreiche randomisierte Studien belegt. In Form des vollständig subkutan implantierbaren Kardioverter-DefibrillatorSystems (S-ICD) besteht die Option, einen entsprechenden Schutz zu gewährleisten und gleichzeitig die mit der transvenös implantierten Elektrode assoziierten Langzeitkomplikationen wie systemische Infektionen und Elektrodendysfunktion zu vermeiden. Mittlerweile ist die Sicherheit und Effektivität des S-ICD durch Ergebnisse großer Registerstudien untermauert worden. Empfehlungen wurden, basierend auf der fehlenden Möglichkeit zur ventrikulären Stimulation, erstmals in die aktuellen
Leitlinien aufgenommen. Perspektivisch wird die Problematik der durch Oversensing bedingten inadäquaten Schockabgaben mittels erweiterter Screening-Methoden und neuer Detektionsalgorithmen wahrscheinlich noch besser kontrolliert werden können. Außerdem scheint durch eine Kombination aus subkutanem ICD und LCP („leadless cardiac pacer") in Zukunft die Kombination antibradykarder und antitachykarder ventrikulärer Stimulation realistisch.

Schlüsselwörter

Defibrillatorsysteme · Plötzlicher Herztod . Primärprophylaxe · Patientenselektion . Komplikationen

\section{Subcutaneous implantable cardioverter-defibrillator. Current status and perspectives}

\section{Abstract}

The use of transvenous implantable cardioverter-defibrillators (ICD) for the prevention of sudden cardiac death has been proven in numerous randomized trials. By using a totally subcutaneous ICD (S-ICD) system, it is expected to ensure appropriate protection while avoiding long-term complications associated with transvenous leads, such as systemic infection and electrode dysfunction. Meanwhile, the safety and effectiveness of the S-ICD has been substantiated by results of large registry studies. Based on the missing option for ventricular stimulation, corresponding recommendations have been integrated into current guidelines for certain patient populations. In the future, the issue of inadequate shocks caused by oversensing may be solved using advanced screening tools and new detection algorithms. Furthermore, a combination of subcutaneous ICD and LCP (leadless cardiac pacer) seems realistic to enable antibradycardia and antitachycardia ventricular pacing.

\section{Keywords}

Defibrillator systems - Sudden cardiac death . Primary prophylaxis . Patient selection . Complications
Anomalien oder Thrombosen nicht möglich ist, besteht in der aktuellen AHALeitlinie eine Klasse-I-Empfehlung für den S-ICD. In der ESC-Leitlinie stehen außerdem junge Patienten im Fokus, bei denen im Hinblick auf die zu erwartende langjährige ICD-Therapie bei transvenöser Elektrodenlage mit entsprechenden Komplikationen im Langzeitverlauf $\mathrm{zu}$ rechnen ist (Empfehlungsgrad IIb). Weiterhin bietet sich der S-ICD besonders für einen Einsatz im Rahmen der Primärprophylaxe des plötzlichen Herztods auch bei strukturellen Herzerkrankungen an.
Bei Patienten, die ein erhöhtes Risiko für den plötzlichen Herztod aufgrund einer hochgradig reduzierten linksventrikulären Ejektionsfraktion (LVEF) im Rahmen einer ischämischen oder nichtischämischen Kardiomyopathie aufweisen, vergehen durchschnittlich $19 \pm 30,9$ Monate bis zur ersten behandlungspflichtigen Kammertachykardie [16]. Betrachtet man die Daten der Meilenstein-Studien zur ICD-Primärprophylaxe, so lag die Rate ventrikulärer Arrhythmien im MADIT-II-Kollektiv in 20 Monaten bei $20 \%$, in der SCD-HeFT-Studie in $45 \mathrm{Mo-}$ 
Die Sicherheit und Wirksamkeit des S-ICD-Systems wird von klinischen Daten und der Erfahrung aus 15 Jahren gestützt, mit über 4000 in abgeschlossenen oder laufenden Studien registrierten Patienten (Pat.).
UNTOUCHED9 - Registrierung von 2000 Pat.

Post-Approval Study ${ }^{8}-1600$ Pat.

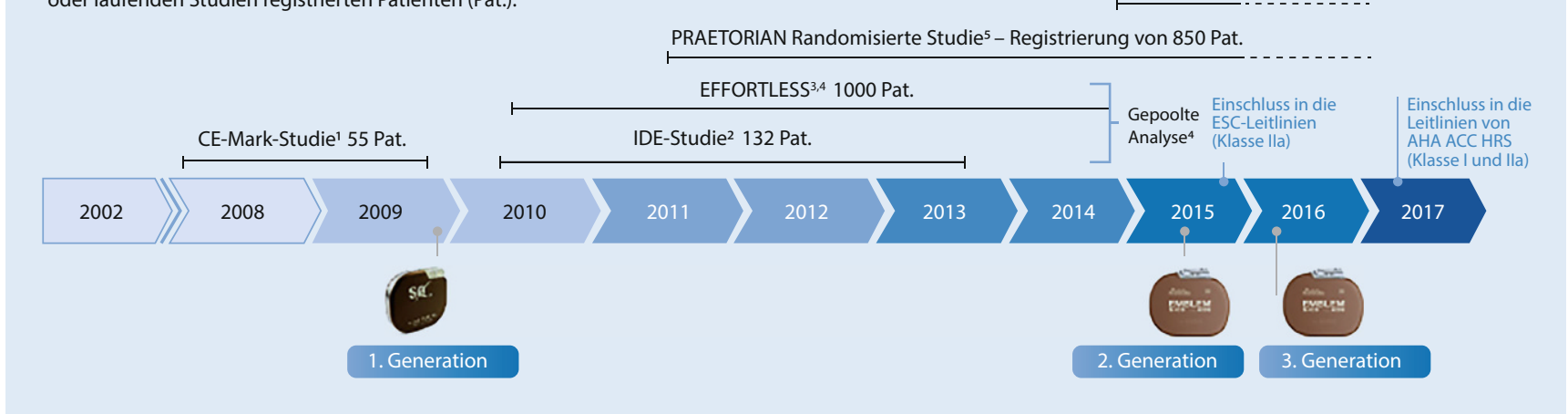

Abb. 3 A Klinische Studien zur S-ICD-Therapie im Zeitstrahl. S-ICD subkutan implantierbarer Kardioverter-Defibrillator. (Abdruck mit freundl. Genehmigung von Boston Scientific)

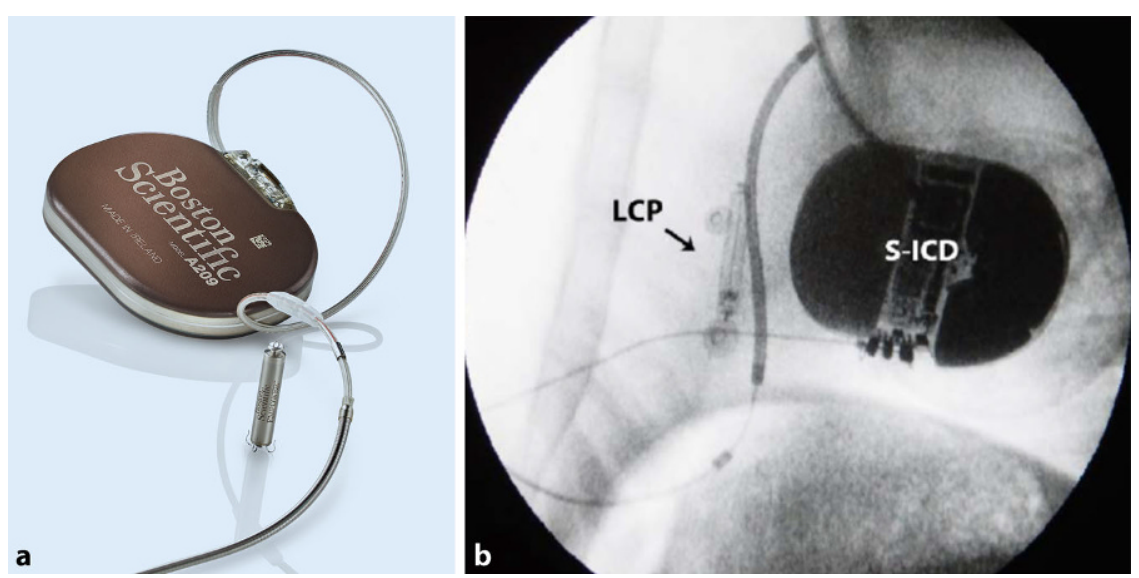

Abb. $4 \Delta$ a Kombination von subkutanem ICD und LCP („leadless cardiac pacemaker"). b Darstellung im Röntgenbild. S-ICD subkutan implantierbarer Kardioverter-Defibrillator. (a Abdruck mit freundl. Genehmigung von Boston Scientific)

naten bei $22 \%[17,18]$. Deshalb scheint ggf. selbst bei im Verlauf möglicherweise auftretenden Kammertachykardien eine initiale S-ICD-Implantation mit möglicher Umrüstung auf ein transvenöses System im Verlauf sinnvoll, um die mit dem herkömmlichen System assoziierten Komplikationen zumindest initial zu vermeiden.

\section{Aktuelle Datenlage zur Sicherheit und Effektivität}

Nach der initialen Zulassungsstudie wurden die ersten Ergebnisse größerer Patientenkollektive in der Pooled-AnalysisStudie, einer kombinierten Analyse der Ergebnisse der IDE-Studie aus dem Jahr 2009 sowie der bis dahin zur Verfügung stehenden Daten des seit 2009 geführ- ten EFFORTLESS-Registers (Evaluation of factors impacting clinical outcome and cost effectiveness of the S-ICD) veröffentlicht (- Abb. 3; [19]). Bei 59 der insgesamt 882 untersuchten Patienten traten dabei in der Nachbeobachtungsphase von durchschnittlich 651 Tagen kumulativ 111 ventrikuläre Arrhythmien auf, von denen 90,1\% mit der ersten Schockabgabe und 98,2\% im Rahmen der insgesamt 5 zur Verfügung stehenden Schocks terminiert werden konnten. 2017 folgte die Veröffentlichung der EFFORTLESSStudie, die anhand von Registerdaten aus der aktuell größten S-ICD-Datenbank die Sicherheit einer S-ICD-Therapie in Bezug auf Komplikationen und inadäquate Schockabgaben bei 985 Patienten in einem Nachbeobachtungszeitraum von durchschnittlich 3,1 Jahren nach Implan- tation untersucht hat [20]. Insgesamt war dabei im Verlauf die Terminierung von Kammerflimmern bzw. ventrikulärer Tachykardien in 97,4\% der Fälle erfolgreich. Im Rahmen von Testungen konnte nach Induktion von Kammerflimmern dieventrikuläre Arrhythmie in nahezu jedem Fall terminiert werden. Durch Umpositionierung des Aggregats mit oder ohne Elektrode konnte auch in 16 von 19 Fällen, in denen die Testung initial nicht erfolgreich war, im Verlauf eine Terminierung des induzierten Kammerflimmers durch den S-ICD erfolgen.

\section{Komplikationen einer S-ICD-Therapie}

Die EFFORTLESS-Studie hat ein besonderes Augenmerk auf mögliche Komplikationen einer S-ICD-Therapie geworfen. Die häufigsten, relativ früh nach Implantation auftretenden Komplikationen waren T-Wellen-Oversensing mit inadäquater Schockabgabe $(1,1 \%)$ sowie unzureichender Tragekomfort, welcher durch das an der linken Brustseite gelegene und im Vergleich zu herkömmlichen Systemen relativ große Aggregat verursacht wurde $(0,8 \%)$.

Besonders hervorzuheben ist, dass die 1-Jahres-Komplikationsrate mit der Zeit bei zunehmender Erfahrung seitens der Implanteure deutlich sank. Im Vergleich zu herkömmlichen transvenösen Systemen war die Häufigkeit von Komplikationen ungefähr vergleichbar, wobei elektrodenassoziierte Probleme 
beim herkömmlichen System signifikant häufiger auftraten [21, 22]. In den 3,1 Jahren der Nachbeobachtungsphase musste bei insgesamt 24 Patienten $(2,4 \%)$ aufgrund von lokalen Infektionen die Explantation des Systems erfolgen; meistens traten die Infektionen dabei im ersten Jahr nach Implantation auf. Systemische Infektionen, wie sie beim transvenösen System in 1-7\% der Fälle vorkommen [23], wurden nicht beobachtet. Bei insgesamt 13 Patienten $(1,3 \%)$ war aufgrund der Indikation zur ATP-Therapie, Resynchronisationstherapie bzw. antibradykarden Stimulation (lediglich 1 Patient) im Verlauf die Umrüstung auf ein transvenöses System erforderlich. Addiert man die Zahl der Patienten, bei denen aufgrund einer Indikation zur ATP-Therapie der Wechsel auf ein transvenöses System erfolgte, mit der Zahl der Patienten, die mehrere Schockabgaben seitens des subkutanen ICD aufgrund rezidivierender monomorpher ventrikulärer Tachykardien erhielten, so hätten bei einer geschätzten ATP-Erfolgsrate von $42 \%$ [24] initial ca. $0,4 \%$ der Patienten von einem konventionellen System mit der Möglichkeit zur ATP-Abgabe profitiert. Im Verlauf der Nachbeobachtungsphase erhielten $11,7 \%$ der Patienten meistens aufgrund von Oversensing der T-Welle einen inadäquaten Schock, seltener war ein nichtkardiales Oversensing bei elektromagnetischer Interferenz dafür verantwortlich. Vergleicht man die Inzidenz von inadäquaten Schockabgaben in der Literatur, so ist diese ungefähr vergleichbar mit der herkömmlicher ICD-Systeme [25-27]. Es deutet sich aber bereits an, dass durch neue Detektionsalgorithmen in der aktuellen S-ICDGeneration (EMBLEM ${ }^{\mathrm{st}} \mathrm{S}$-ICD) die Rate an durch T-Wellen-Oversensing verursachten inadäquaten Schockabgaben deutlich reduziert werden kann [28]. Zukünftige Daten noch laufender Studien, wie die der PRAETORIAN-Studie (A prospective, randomized comparison of subcutaneous and transvenous implantable cardioverter defibrillator therapy) werden sicher weiteren Aufschluss über Effektivität, Sicherheit und Komplikationsrate von subkutanen ICD-
Systemen im Vergleich zu transvenösen Systemen geben [29].

\section{Ausblick}

Aktuell stellt die fehlende Möglichkeit zur antibradykarden Stimulation und ATP-Abgabe den entscheidenden limitierenden Faktor der S-ICD-Therapie dar. Prinzipiell können diese Funktionen aber durch Kombination von subkutanem ICD und LCP (,leadless cardiac pacemaker") ermöglicht werden (- Abb. 4). In diesem Zusammenhang zeigte eine 2016 veröffentliche Studie an Schafen, denen jeweils ein subkutaner ICD sowie ein LCP-Prototyp (Boston Scientific) in die Spitze des rechten Ventrikels implantiert wurde, vielversprechende neue Perspektiven auf. Mittels eines im linken Ventrikel platzierten Stimulationskatheters wurden ventrikuläre Tachykardien induziert [30]. Dabei konnte gezeigt werden, dass die intrakorporale unidirektionale Kommunikation zwischen S-ICD und LCP einwandfrei funktionierte und so durch den S-ICD getriggerte ATP-Abgaben seitens des LCP möglich waren.

\section{Fazit für die Praxis}

- Trotz guter Studiendaten und steigender Implantationszahlen macht der S-ICD weiterhin einen eher geringen Teil der gesamten ICDImplantationen in Deutschland aus.

- Laut aktuellen Leitlinien eignet sich der S-ICD besonders für Patienten ohne ventrikulären Stimulationsbedarf bzw. für junge Patienten mit Kammerflimmern.

- Auch für die Primärprophylaxe bei hochgradig reduzierter linksventrikulärer Funktion wird das System immer häufiger verwendet.

- In der Sekundärprophylaxe sollte bei dokumentierten monomorphen ventrikulären Tachykardien weiterhin auf ein transvenöses System zurückgegriffen werden, allerdings scheint die Möglichkeit einer ATP-Abgabe ohne transvenöse Elektrodenlage durch Kombination von S-ICD und LCP realistisch.
- Für das Screening steht ein automatisches und ein manuelles Tool zur Verfügung.

- Durch T-Wellen-Oversensing verursachte inadäquate Schockabgaben können durch neue Detektionsalgorithmen reduziert und in Zukunft wahrscheinlich besser vermieden werden.

\section{Korrespondenzadresse}

\section{Philipp Niehues}

Klinik für Kardiologie II - Rhythmologie, Universitätsklinikum Münster, Albert-

Schweitzer Campus 1

48149 Münster, Deutschland

philipp.niehues@ukmuenster.de

Förderung. Die Open-access-Gebühren wurden von der Firma Boston Scientific übernommen.

\section{Einhaltung ethischer Richtlinien}

Interessenkonflikt. P. Niehues gibt an, dass kein Interessenkonflikt besteht. G. Frommeyer, F. Reinke und L. Eckardt haben Vortragshonorare von Boston Scientific erhalten.

Dieser Beitrag beinhaltet keine von den Autoren durchgeführten Studien an Menschen oder Tieren.

Open Access Dieser Artikel wird unter der Creative Commons Namensnennung 4.0 International Lizenz (http://creativecommons.org/licenses/by/4.0/deed. de) veröffentlicht, welche die Nutzung, Vervielfältigung, Bearbeitung, Verbreitung und Wiedergabe in jeglichem Medium und Format erlaubt, sofern Sie den/die ursprünglichen Autor(en) und die Quelle ordnungsgemäßnennen, einen Linkzur Creative Commons Lizenz beifügen und angeben, ob Änderungen vorgenommen wurden.

\section{Literatur}

1. Bardy GH et al (2010) An entirely subcutaneous implantable cardioverter-defibrillator. N Engl J Med 363(1):36-44

2. Markewitz A (2018) Jahresbericht 2015 des Deutschen Herzschrittmacher- und Defibrillatorregisters, Teil 2 - Implantierbare KardioverterDefibrillatoren (ICD): Fachgruppe Herzschrittmacher beim IQTIG - Institut für Qualitätssicherung und Transparenz im Gesundheitswesen Bd. 29

3. van Rees JB et al (2011) Implantation-related complications of implantable cardioverter-defibrillators and cardiac resynchronization therapy devices: a systematic review of randomized clinical trials. J Am Coll Cardiol 58(10):995-1000

4. Sohail MRetal (2011) Mortality and cost associated with cardiovascular implantable electronic device infections. Arch Intern Med 171(20):1821-1828

5. Maisel WH, Kramer DB (2008) Implantable cardioverter-defibrillator lead performance. Circulation 117(21):2721-2723 
6. Zumhagen S et al (2012) Totally subcutaneous implantable cardioverter defibrillator with an alternative, right parasternal, electrode placement. Pacing Clin Electrophysiol 35(9):e254-7

7. Bettin $M$ et al (2015) Recent advances in the entirely subcutaneous ICD System. JACC Clin Electrophysiol 7:46

8. Bettin M et al (2018) Change of sensing vector in the subcutaneous ICD during follow-up and after device replacement. J Cardiovasc Electrophysiol. https://doi.org/10.1111/jce.13647

9. Frommeyer $\mathrm{G}$ et al (2016) Intraoperative defibrillation testing of subcutaneous implantable cardioverter-defibrillator systems-A simple issue? J Am Heart Assoc 5(3):e3181

10. Moss AJ et al (2012) Reduction in inappropriate therapy and mortality through ICD programming. NEngl J Med 367(24):2275-2283

11. Bettin M et al (2018) Follow-up of the first patients with a totally subcutaneous ICD in Germany from implantation till battery depletion. Clin Res Cardiol. https://doi.org/10.1007/s00392-018-1296-1

12. Bogeholz $\mathrm{N}$ et al (2018) Direct comparison of the novel automated screening tool (AST) versus the manual screening tool (MST) in patients with already implanted subcutaneous ICD. Int J Cardiol 265:90-96

13. Priori SG et al (2015) ESC Guidelines for the management of patients with ventricular arrhythmias and the prevention of sudden cardiac death: The Task Force for the Management of Patients with Ventricular Arrhythmias and the Prevention of Sudden Cardiac Death of the European Society of Cardiology (ESC). Endorsed by: Association for European Paediatric and Congenital Cardiology (AEPC). Eur Heart J36(41):2793-2867

14. Al-Khatib SM et al (2017) AHA/ACC/HRS Guideline for Management of Patients With Ventricular Arrhythmias and the Prevention of Sudden Cardiac Death: A Report of the American College of Cardiology/American Heart Association TaskForce on Clinical Practice Guidelines and the Heart Rhythm Society. Circulation. https://doi.org/10. 1161/CIR.0000000000000548

15. de Bie MK et al (2013) Suitability for subcutaneous defibrillator implantation: results based on data from routine clinical practice. Heart 99(14):1018-1023

16. Streitner F et al (2011) Comparison of ventricular tachyarrhythmia characteristics in patients with idiopathic dilated or ischemic cardiomyopathy and defibrillators implanted for primary prevention. Clin Cardiol 34(10):604-609

17. Moss AJ et al (2002) Prophylactic implantation of a defibrillator in patients with myocardial infarction and reduced ejection fraction. N Engl J Med 346(12):877-883

18. Bardy GH et al (2005) Amiodarone or an implantable cardioverter-defibrillator for congestive heart failure. N Engl J Med 352(3):225-237

19. Burke MC et al (2015) Safety and efficacy of the totally subcutaneous implantable defibrillator. J Am Coll Cardiol 65(16):1605-1615

20. Boersma $L$ et al (2017) Implant and midterm outcomes of the subcutaneous implantable cardioverter-defibrillator registry: the EFFORTLESS study. J Am Coll Cardiol 70(7):830-841

21. Brouwer TF et al (2016) Long-term clinical outcomes of subcutaneous versus transvenous implantable defibrillator therapy. J Am Coll Cardiol 68(19):2047-2055

22. Köbe J et al (2013) Implantation and follow-up of totally subcutaneous versus conventional im- plantable cardioverter-defibrillators: a multicenter case-control study. Heart Rhythm 10(1):29-36

23. Uslan DZ et al (2007) Permanent pacemaker and implantable cardioverter defibrillator infection: a population-based study. Arch Intern Med 167(7):669-675

24. Wathen MS et al (2004) Prospective randomized multicenter trial of empirical antitachycardia pacing versus shocks for spontaneous rapid ventricular tachycardia in patients with implantable cardioverter-defibrillators: Pacing Fast Ventricular Tachycardia Reduces Shock Therapies (PainFREE Rx II) trial results. Circulation 110(17):2591-2596

25. Daubert JP et al (2008) Inappropriate implantable cardioverter-defibrillator shocks in MADIT II: frequency, mechanisms, predictors, and survival impact. J Am Coll Cardiol 51(14):1357-1365

26. Saxon LA et al (2010) Long-term outcome after ICD and CRT implantation and influence of remote device follow-up. Circulation 122(23):2359-2367

27. Poole JE et al (2008) Prognostic importance of defibrillator shocks in patients with heart failure. NEngl J Med 359(10):1009-1017

28. Theuns DA et al (2016) 56-44: Evaluation of a High Pass Filter Designed to Reduce Oversensing in the S-ICD. Europace 18(suppl_1):i42-i42

29. Nordkamp OLRA et al (2012) Rationale and design of the PRAETORIAN trial: a prospective, RAndomizEd comparison of subcuTaneOus and tRansvenous ImplANtable cardioverter-defibrillator therapy. Am Heart J 163(5):753-760.e2

30. Tjong FVY et al (2016) Communicating Antitachy cardia Pacing-Enabled Leadless Pacemaker and Subcutaneous Implantable Defibrillator. J Am Coll Cardiol 67(15):1865-1866 